\title{
A preliminary study of the clinical application of optic pathway diffusion tensor tractography in suprasellar tumor surgery: preoperative, intraoperative, and postoperative assessment
}

\author{
Mohamadreza Hajiabadi, MD, PhD, ${ }^{1-3}$ Madjid Samii, MD, PhD,1 and Rudolf Fahlbusch, MD, PhD'1 \\ ${ }^{1}$ International Neuroscience Institute, Hannover, Germany; ${ }^{2}$ Brain and Spinal Cord Injury Research Center, Neuroscience \\ Institute, and ${ }^{3}$ Iranian International Neuroscience Institute, Tehran University of Medical Sciences, Tehran, Iran
}

\begin{abstract}
OBJECTIVE Visual impairments are the most common objective manifestations of suprasellar lesions. Diffusion tensor imaging (DTI) is a noninvasive MRI modality that depicts the subcortical white matter tracts in vivo. In this study the authors tested the value of visual pathway tractography in comparison with visual field and visual acuity analyses.

METHODS This prospective study consisted of 25 patients with progressive visual impairment due to suprasellar mass lesions and 6 control patients with normal vision without such lesions. Visual acuity, visual field, and the optic fundus were examined preoperatively and repeated 1 week and 3 months after surgery. Visual pathway DTI tractography was performed preoperatively, intraoperatively immediately after tumor resection, and 1 week and 3 months after surgery.

RESULTS In the control group, pre- and postoperative visual status were normal and visual pathway tractography revealed fibers crossing the optic chiasm without any alteration. In patients with suprasellar lesions, vision improved in 24 of 25 . The mean distance between optic tracts in tractography decreased after tumor resection and detectable fibers crossing the optic chiasm increased from $12 \%$ preoperatively to $72 \%$ postoperatively 3 months after tumor resection, and undetectable fibers crossing the optic chiasm decreased from $88 \%$ preoperatively to $27 \%$ postoperatively 3 months after tumor resection. Visual improvement after tumor removal 1 week and 3 months after surgery was significantly correlated with the distance between optic tracts in intraoperative tractography $(p<0.01)$.
\end{abstract}

CONCLUSIONS Visual pathway DTI tractography appears to be a promising adjunct to the standard clinical and paraclinical visual examinations in patients with suprasellar mass lesions. The intraoperative findings, in particular the distance between optic tract fibers, can predict visual outcome after tumor resection. Furthermore, postoperative application of this technique may be useful in following anterior optic pathway recovery.

http://thejns.org/doi/abs/10.3171/2015.6.JNS1546

KEY WORDS diffusion tensor imaging; visual impairment score; optic pathway tractography; suprasellar tumor; diagnostic and operative techniques

$\mathrm{O}$ PTIC nerves, the optic chiasm, and optic tracts, which are located in the suprasellar region, can be damaged anatomically and functionally by tumors in this area. Due to this anatomical relationship, visual impairment is the most common objective manifestation of such tumors..$^{10}$ The severity of visual loss (acuity, field, and color discrimination) depends on the tumor's characteristic. Large, firm, and capsular tumors appear to lead to more severe loss of vision. ${ }^{6}$ On the other hand, visual acuity and visual field examination reflect the degree of optic pathway compression. This examination requires the cooperation of the patient; therefore in some critical situations such as an intubated patient, a noncooperative state, and pediatric patients, decision making while considering the ophthalmological examination is not possible. Furthermore, there is a continuing interest to evaluate vi-

ABBREVIATIONS DTI = diffusion tensor imaging; FA = fractional anisotropy; $\mathrm{HARDI}=$ high angular resolution diffusion imaging; $\mathrm{MD}=$ mean diffusivity; $\mathrm{MFL}=$ minimum fiber length; ROI = region of interest; TSSS = transsphenoidal sinus surgery; VIS = visual impairment score.

SUBMITTED January 8, 2015. ACCEPTED June 30, 2015.

INCLUDE WHEN CITING Published online December 18, 2015; DOI: 10.3171/2015.6.JNS1546. 
sual function intraoperatively during surgery around the visual pathways. ${ }^{18}$ Diffusion tensor imaging (DTI) is a noninvasive MRI modality that depicts the probable location and geometrical organization of subcortical tracts in vivo. ${ }^{17}$ Displacement, edema, and microstructural damage of tracts can be evaluated by DTI,${ }^{23}$ but the validity and reliability of these techniques, especially in the visual pathway system, are not yet clear. ${ }^{12,14,26}$ Visual pathway fiber tractography may serve as a way to broaden our anatomical-functional understanding and provide better visualization of the optic pathway. In this preliminary study, we attempted to define preoperative, intraoperative, and postoperative alterations of the visual pathway fibers in 25 patients with suprasellar tumors using DTI tractography, and to correlate the DTI findings with the clinical visual status of the patients. The optic pathway was evaluated anatomically (location and structure) with DTI tractography and functionally with ophthalmological examination of visual acuity and visual fields. We compared DTI and clinical findings preoperatively, intraoperatively, and postoperatively. There have been some studies that reported on the posterior visual pathways in brain tumor surgery, ${ }^{20,22,28}$ and also some recent studies concerning the anterior visual pathways, ${ }^{1,2,13,27}$ but none involving intraoperative DTI. To our knowledge, this is the first report to study the anterior visual pathway using intraoperative DTI tractography in relation to ophthalmological examination for surgical treatment of suprasellar lesions.

\section{Methods}

Between April 2013 and October 2014, 25 successive patients underwent operations at the International Neuroscience Institute in Hannover, Germany, for visual disturbance due to compression of the optic chiasm. Six patients with normal vision and no visual pathway compressive lesions were included as the control group in our prospective pilot study. The control group consisted of patients who underwent operations because of other pathologies. All patients and control subjects were examined by 1 ophthalmologist. Visual acuity, funduscopy, intraocular pressure, and visual fields were evaluated preoperatively and then 1 week and 3 months after surgery. Visual acuity was determined by the Snellen chart and visual field defects were assessed by the Goldmann and Humphrey field analyzer. These ophthalmological findings were analyzed according to the guidelines of the German Ophthalmological Society. The scores of visual acuity and visual field defects were added together for each patient, and provided the visual impairment score (VIS). This scoring and evaluation of pituitary hormones was performed for the patients with hypothalamus-pituitary lesions preoperatively, and 1 week and 3 months after surgery, as detailed in a previous publication. ${ }^{7}$ Before surgery, all patients gave their informed consent for intraoperative MRI-DTI investigation to evaluate the visual pathway. The patients with suprasellar mass lesions underwent a direct, microscopic, endonasal transsphenoidal approach (endoscope assisted) or transcranial (frontolateral) approach. The lesions were removed microsurgically with the assistance of neuronavigation (Vector Vision 2; BrainLab), a Storz endoscope, and intraoperative MRI.

\section{Visual Pathway DTI Tractography}

All patients underwent 1.5-T brain MRI with and without contrast preoperatively, intraoperatively, and twice (1 week and 3 months) after surgery. The MRI scanner used was a Magnetom Espree (Siemens AG Medical Solutions) with a superconductive 1.5-T magnet. A magnetization prepared rapid-acquisition gradient-echo sequence with DTI was obtained. DTI resolution was $128 \times 128 \times 60$ voxels and voxel size was $1.875 \times 1.875 \times 9 \mathrm{~mm}$. DTI was performed in 20 directions, with 3 average images, with $b$ values of $1000 \mathrm{sec} / \mathrm{mm}^{2}$. For each data set, fiber tractography was performed using commercially available software (BrainLAB). Seed regions were placed at the optic chiasm, occipital cortex (pericalcarine and precuneus regions), and deep regions between the superior and middle temporal gyri lateral to the trigone for each side. ${ }^{22}$ Tracking was performed with a fractional anisotropy (FA) threshold of 0.01 and minimum fiber length (MFL) of $5 \mathrm{~mm}$.

\section{Statistical Analysis}

All data analyses were conducted using SPSS (version 16.0 for Windows, SPSS Inc.). Repeated-measures ANOVA was used to compare the VIS and distance between optic tract means at specific time points (preoperatively, intraoperatively, and 1 week and 3 months after surgery). Pearson's correlation coefficients were applied to examine the relationship between the distance of the optic tracts in visual pathway tractography and the change in VIS after decompression of the optic chiasm, and an independent $\mathrm{t}$-test was used to compare the VIS difference with detectable or undetectable fibers crossing the optic chiasm. All statistical tests were 2 -sided and $\mathrm{p}<0.05$ was considered statistically significant.

\section{Results}

The mean age of the 25 patients with compression of the optic chiasm was $53.08 \pm 18.61$ years (range 11-87 years), 15 were male $(60 \%), 18$ had pituitary adenoma (72\%), and in 22 a transsphenoidal sinus surgery (TSSS) approach was performed (Table 1). The visual status improved in all patients postoperatively except in 1, whose vision deteriorated because of optic nerve compression due to suprasellar bleeding that underwent reoperation.

In the control group (6 cases), preoperative and postoperative visual examination showed normal visual status. Visual pathway tractography in this group performed preoperatively and postoperatively revealed fibers crossing the optic chiasm without any alteration. In our patients $(25$ cases), the mean VIS changed from $28.32 \pm 18.15$ preoperatively to $20.76 \pm 17.46$ at 1 week and $14.04 \pm 14.98$ at 3 months after surgery. Using an ANOVA, estimated mean VIS showed a significant decrease $(\mathrm{p}<0.001$; Fig. 1).

The mean distance between optic tracts in tractography changed from $9.46 \pm 7.26$ preoperatively to $5.97 \pm 6.21$ intraoperatively, to $4.84 \pm 6.48$ at 1 week after surgery, and then $1.90 \pm 4.14$ at 3 months after surgery. Using an ANOVA, the estimated mean distance between optic tracts in tractography showed a significant decrease $(\mathrm{p}<$ 0.001; Fig. 2).

Preoperative tractography in $22(88 \%)$ of 25 patients 
TABLE 1. Patient demographics and clinical characteristics

\begin{tabular}{cc}
\hline \multicolumn{1}{c}{ Variable } & Value \\
\hline Mean age (yrs) & $53.08 \pm 18.61$ \\
\hline Sex (\%) & $15(60)$ \\
\hline Male & $10(40)$ \\
\hline Female & $22(88)$ \\
\hline Type of surgery (\%) & $3(12)$ \\
\hline TSSS & $18(72)$ \\
\hline Transcranial surgery & $2(8)$ \\
\hline Pathology (\%) & $3(12)$ \\
\hline Pituitary adenoma & $2(8)$ \\
\hline Meningioma &
\end{tabular}

showed no crossing fibers in the optic chiasm (undetectable chiasm). Among these 22 patients, chiasm-crossing fibers were revealed in 5 patients with intraoperative tractography, in 4 patients 1 week after surgery, and in 6 patients 3 months after surgery. After 3 months in $28 \%$ of the patients, the optic chiasm was still undetectable on DTI tractography (Fig. 3).

There was a statistically significant relationship between the detection of chiasm-crossing fibers in tractography 1 week after tumor resection and visual improvement 3 months after the operation $(\mathrm{p}=0.002)$. The distance between optic tracts on intraoperative tractography was significantly inversely correlated with visual improvement 1 week and 3 months after surgery using the Pearson correlation coefficient (correlation coefficients 0.69 and 0.59 , respectively; $\mathrm{p}<0.01)$.

\section{Illustrative Cases}

Case 1

A 46-year-old man presented with progressive visual

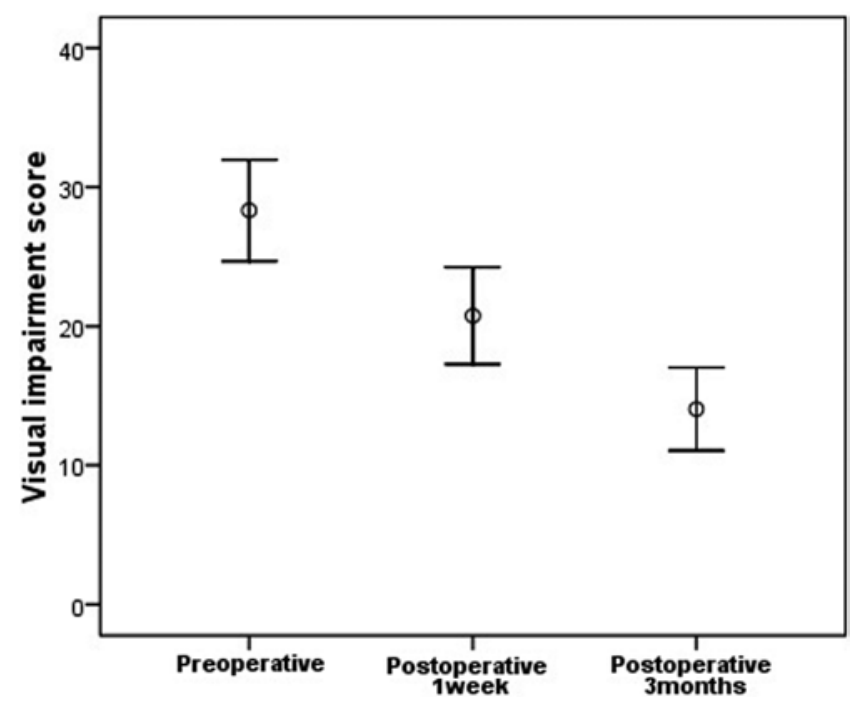

FIG. 1. Graph of the estimated mean VIS preoperatively, 1 week after surgery, and 3 months after surgery in 25 patients with suprasellar tumors.

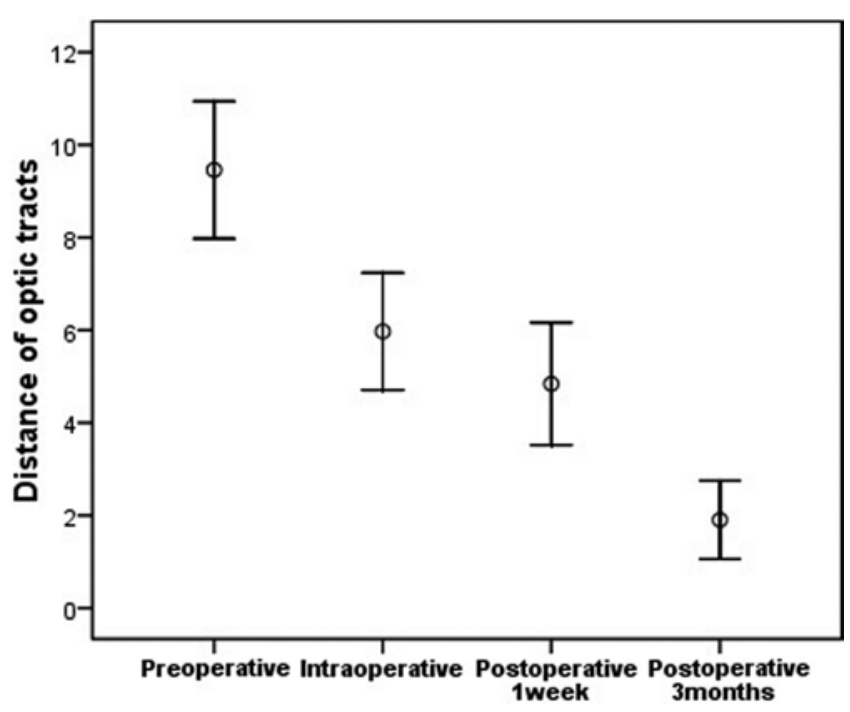

FIG. 2. Graph showing the estimated mean distance between optic tracts on tractography preoperatively, intraoperatively, 1 week after surgery, and 3 months after surgery, in 25 patients with suprasellar tumors.

loss in his left eye over the previous 2 months. He had a history of severe hyponatremia and acute hypocortisolemic symptoms. MRI showed an intrasellar and suprasellar mass with cystic changes, which compressed the optic chiasm. The patient also had secondary hypothyroidism. The tumor was removed completely using a microscopic endonasal transsphenoidal (endoscopic-assisted) approach. The pathological examination showed a nonfunctional pituitary adenoma. The postoperative course of the patient was uneventful. We found more visual fiber tracts and downward location of the chiasm after tumor decompression intraoperatively and postoperatively (Fig. 4).

\section{Case 2}

A 57-year-old woman presented with progressive visual deterioration over the last year. MRI showed a large intra- and suprasellar mass with compression of the optic chiasm. The patient had secondary hypothyroidism and hypogonadism. The tumor-a nonfunctional pituitary adenoma-was completely removed using a microscopic endonasal transsphenoidal (endoscopic-assisted) approach. The postoperative period was uneventful. Intraoperative DTI tractography after tumor decompression showed a downward location and approximation of visual fiber in bilateral optic tracts. Crossing fibers of the optic chiasm appeared 3 months after surgery (Fig. 5).

\section{Discussion}

Optic chiasm compression due to suprasellar mass can cause visual acuity impairment, visual field defects, and color discrimination disturbance. Visual acuity is commonly found to be impaired at an early stage, before any perimetric signs. ${ }^{3}$ In some optic pathway disease such as glaucoma, visual field defects can be detected by automated perimetry, usually after $25 \%-35 \%$ of ganglion cell axonal loss. ${ }^{24}$ Although the degree of visual acuity impairment is correlated with the extent of visual field defect, ${ }^{8,9}$ their response to surgical decompression is not always 


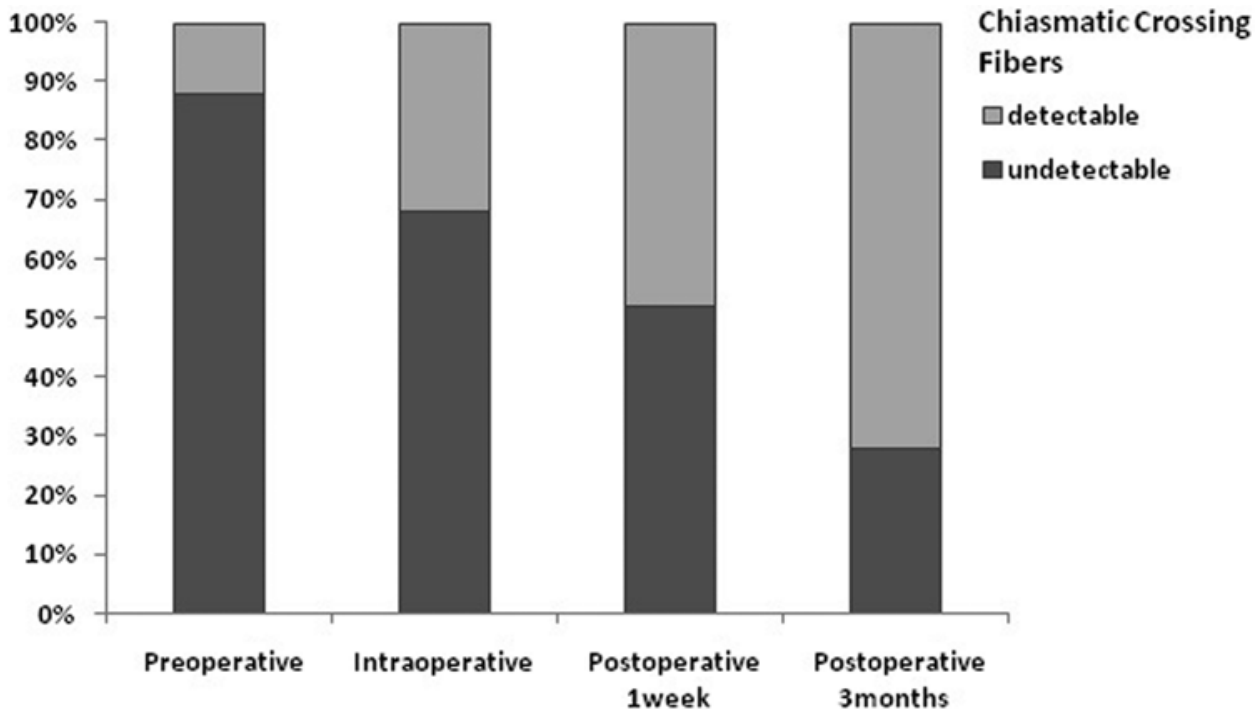

FIG. 3. Bar graph of the percentages of detectable chiasmatic-crossing fibers after tumor resection.

parallel to each other. ${ }^{16}$ Vision improves progressively at least within the first year after transsphenoidal surgery. ${ }^{5}$ The improvement of visual dysfunction after surgical treatment is supposed to consist of two phases..$^{11}$ Early recovery leads to restoration of signal conduction and delayed recovery related to restoration of axonal transport and remyelination. ${ }^{11}$ In the early phase, more than $50 \%$ of the eventual recovery takes place within the first 3 months after surgery. ${ }^{27}$ Our findings showed that the visual improvement noted during the first postoperative week was more than what was observed between 1 week and 3 months after surgery.

Although MRI is the traditional imaging modality for optic nerves, the optic chiasm, and proximal of optic tracts, ${ }^{15}$ using this method we cannot distinguish the microstructural changes of the nerve fibers. Furthermore, in some invasive unresectable pituitary macroadenomas, preservation of vision during the surgery is more important than complete tumor resection; therefore the importance of an intraoperative imaging method to evaluate the anatomical and/or functional characteristics of the visual pathway is very relevant. DTI evaluates diffusion in multiple, different directions (represented by vectors with magnitude and direction). ${ }^{12}$ DTI fiber tractography is a mathematical technique to reconstruct the white matter tracts in a 3D view. Microstructural damage due to nerve fiber compression may affect DTI quantitative measures such as mean diffusivity (MD), apparent diffusion coefficient, and FA. ${ }^{23,25}$ Nerve fiber damage due to compression may manifest as lower FA and higher MD in the specific region of interest (ROI) in DTI. Diffusion-weighted imaging is sensitive to motion, therefore these images have lower signal-to-noise ratio and are susceptible to artifacts. ${ }^{14,26}$ The range of tensor-derived measures (MD, FA) change widely, even in a healthy population, and this variability can be related to interindividual biological differences as well as experimental noise. ${ }^{4}$ Air in the sphenoid sinus, CSF flow in the basal cisterns, orbital fat, muscle movement in the orbit, and bleeding are known to produce ar- tifacts on DTI. ${ }^{21,30}$ Therefore, we tracked the fibers with FA and MFL values and ROIs that had the lowest artifact for each patient. The same values were used afterward at repeated intraoperative and postoperative DTI. All visual fibers in DTI tractography were compared with their actual anatomical location on MRI to delete artificial fibers.

Until now, there has not been a published study evaluating the link between DTI findings and clinical visual status. ${ }^{13}$ However, Loeber et al. observed that monocular visual deficits often corresponded to a loss or attenuation of ipsilateral prechiasmatic fibers and postchiasmatic fibers, although this finding was without statistical significance..$^{13}$ They believe that with the application of DTI in imaging studies, lesions might be better described for surgical planning and more accurately classified based on arrangement of native fibers. ${ }^{13}$ Bauer et al., using high angular resolution diffusion imaging (HARDI), showed a significant reduction in association fibers associated with the major pathways implicated in visual processing in 2 patients with cortical visual impairment. ${ }^{2}$ Techniques such as HARDI, Q-ball imaging, and diffusion spectrum imaging have been recently used to better characterize the physiological complex structures with kissing and crossing fibers. Although diffusion spectrum imaging is a valuable technique in research, it is not currently in clinical use. Hardware and time limitations have also precluded widespread use of these 3 techniques. ${ }^{12}$

In our series, the tumor was resected completely in 22 of 25 patients, and in 3 patients subtotal tumor removal was performed. In 1 of these patients, vision deteriorated transiently at 1 week after surgery, and visual pathway tractography at this time showed a transient increased distance between the optic tracts as well (Fig. 6). The chiasmcrossing fibers in $88 \%$ of the patients were not detectable preoperatively even with the lowest FA and MFL, but 3 months after the operation only $28 \%$ of the patients had undetectable chiasm. The reappearance of optic chiasm fibers on tractography 1 week after surgery was associated with a better visual outcome 3 months after surgery $(\mathrm{p}=$ 

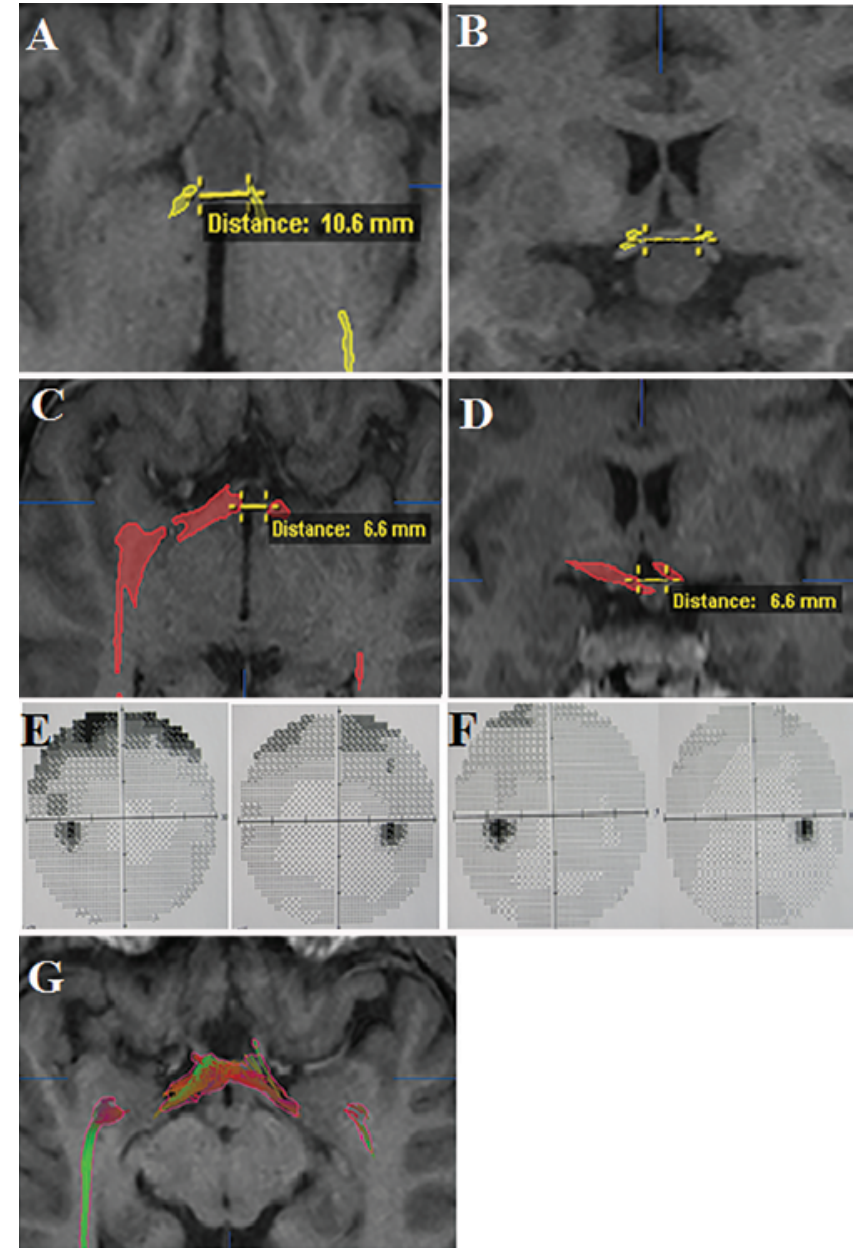

FIG. 4. Preoperative (A, B, and $E$ ), intraoperative ( $C$ and $D)$, and postoperative evaluation ( $F$ and $G$ ). A and B: Preoperative axial and coronal visual pathway tractography in a patient with nonfunctional pituitary adenoma. The distance between the optic tract fibers was $10.6 \mathrm{~mm}$. C and D: Intraoperative coronal visual pathway tractography. The distance between optic tract fibers decreased to $6.6 \mathrm{~mm}$. E: Preoperative visual field perimetry. $\mathbf{F}$ and $\mathbf{G}$ : Visual field perimetry and visual pathway tractography (axial view) 1 week after surgery. Figure is available in color online only.

0.002). Although not statistically significant, a better visual outcome both at 1 week and 3 months postoperatively was observed in patients whose optic chiasm fibers reappeared intraoperatively. In intraoperative tractography, the distance between optic tracts showed an excellent correlation with visual improvement twice after surgery (at 1 week and 3 months). Release of a nerve conduction block after optic pathway decompression causes better axoplasmic transportation and probably manifests as the reappearance of chiasm-crossing nerve fibers or approximation of optic tracts on DTI tractography. This correlation between visual improvement and distance between optic tracts in tractography has also been shown at 3 months after surgery.

Age may have some effect on DTI quantitative measures such as diffusivity, apparent diffusion coefficient, and FA. Recently, Yan et al. showed a correlation between age and range of FA and MD in monkeys ${ }^{29}$ and another
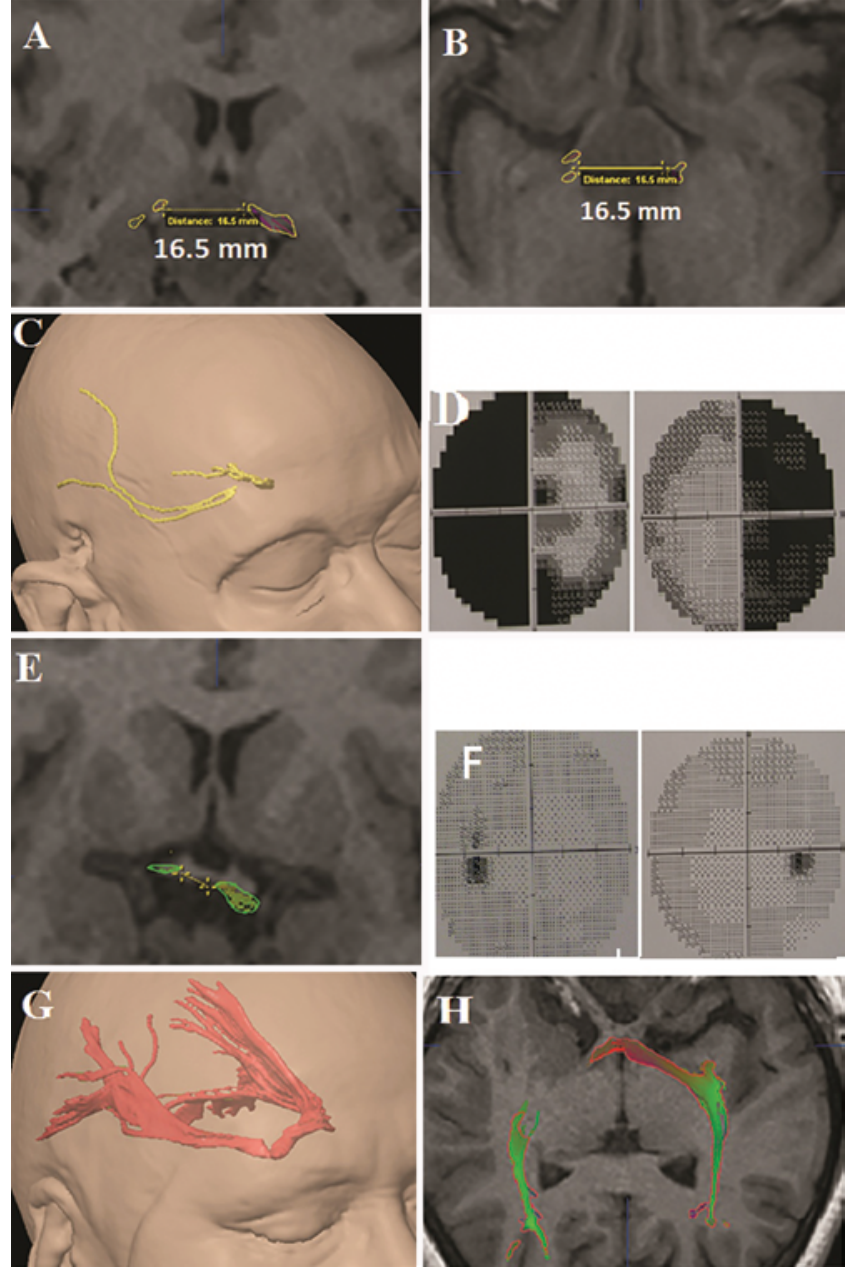

FIG. 5. Preoperative (A-D), intraoperative (E), and postoperative evaluation $(\mathrm{F}-\mathrm{H})$. A-C: Preoperative visual pathway tractography in coronal, axial, and 3D views in a patient with nonfunctional pituitary adenoma. The distance between the optic tracts was $16.5 \mathrm{~mm}$. D: Preoperative visual field defect on visual field perimetry. E: Intraoperative visual pathway tractography after tumor removal showing approximated optic tract fibers. The distance between the fibers decreased to $6.7 \mathrm{~mm}$. F: Visual field 3 months after surgery on visual field perimetry. $\mathbf{G}$ and $\mathrm{H}$ : Visual pathway tractography 3 months after surgery in $3 \mathrm{D}$ and axial views. Figure is available in color online only.

study by Salminen et al. suggested that aging has a significant and differentiated impact on gray/white matter in healthy older adults. ${ }^{19}$ In our experience there was no statistically significant relationship between a patient's age and pathology of the tumor with DTI findings.

\section{Conclusions}

With this pilot study we found a correlation between optic tract distance on visual pathway tractography and visual improvement, at 1 week and 3 months after surgery. Visual pathway DTI tractography appears to be a promising adjunct to the standard clinical and paraclinical visual examinations in patients with suprasellar masses. The intraoperative findings can predict the visual outcome after tumor resection. Furthermore, postoperative applica- 

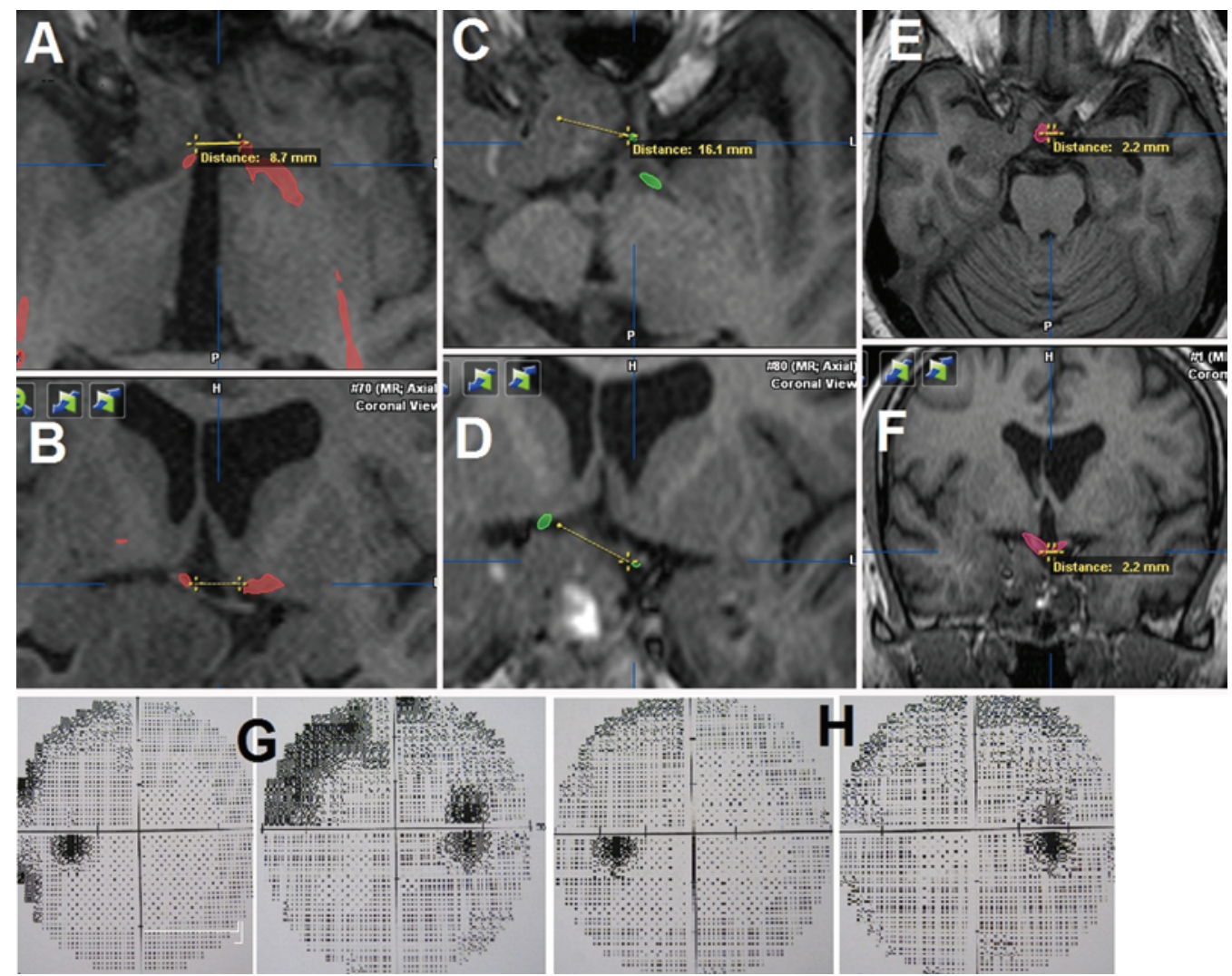

FIG. 6. A and B: Axial and coronal intraoperative optic tract tractography in a patient with a firm pituitary macroadenoma. C and D: Axial and coronal postoperative visual pathway tractography 1 week after surgery; the distance between the optic tracts increased to $16.1 \mathrm{~mm}$. E and F: Axial and coronal postoperative visual pathway tractography 3 months after surgery; the distance between the optic tracts decreased to $2.2 \mathrm{~mm}$. G: Postoperative visual field perimetry showing the visual field deteriorated 1 week after surgery. H: Visual field perimetry showing improvement of the visual field 3 months after surgery. Figure is available in color online only.

tion of this technique may be useful in detecting anterior optic pathway recovery in patients. As a further step, better software for nerve fiber tractography should be developed in cooperation with computer scientists to produce better objective visualization of optic pathway fibers.

\section{Acknowledgments}

We would like to express our great appreciation to Dr. Fariba Mohammadi Rad, Department of Ophthalmology, and Dr. Elolf, Department of Neuroradiology, at the International Neuroscience Institute hospital for their excellent cooperation.

\section{References}

1. Anik I, Anik Y, Koc K, Ceylan S, Genc H, Altintas O, et al: Evaluation of early visual recovery in pituitary macroadenomas after endoscopic endonasal transphenoidal surgery: Quantitative assessment with diffusion tensor imaging (DTI). Acta Neurochir (Wien) 153:831-842, 2011

2. Bauer CM, Heidary G, Koo BB, Killiany RJ, Bex P, Merabet LB: Abnormal white matter tractography of visual pathways detected by high-angular-resolution diffusion imaging (HARDI) corresponds to visual dysfunction in cortical/cerebral visual impairment. J AAPOS 18:398-401, 2014

3. Berman JI, Glass HC, Miller SP, Mukherjee P, Ferriero DM, Barkovich AJ, et al: Quantitative fiber tracking analysis of the optic radiation correlated with visual performance in premature newborns. AJNR Am J Neuroradiol 30:120-124, 2009

4. Carlson HL, Laliberté C, Brooks BL, Hodge J, Kirton A, Bello-Espinosa L, et al: Reliability and variability of diffusion tensor imaging (DTI) tractography in pediatric epilepsy. Epilepsy Behav 37:116-122, 2014

5. Dekkers OM, de Keizer RJ, Roelfsema F, Vd Klaauw AA, Honkoop PJ, van Dulken H, et al: Progressive improvement of impaired visual acuity during the first year after transsphenoidal surgery for non-functioning pituitary macroadenoma. Pituitary 10:61-65, 2007

6. Fahlbusch R, Marguth F: Optic nerve compression by pituitary adenomas, in Samii M, Jannetta PJ (eds): The Cranial Nerves. Vienna: Springer, 1981, pp 140-147

7. Fahlbusch R, Schott W: Pterional surgery of meningiomas of the tuberculum sellae and planum sphenoidale: surgical results with special consideration of ophthalmological and endocrinological outcomes. J Neurosurg 96:235-243, 2002

8. Findlay G, McFadzean RM, Teasdale G: Recovery of vision following treatment of pituitary tumours; application of a new system of assessment to patients treated by transsphenoidal operation. Acta Neurochir (Wien) 68:175-186, 1983

9. Gnanalingham KK, Bhattacharjee S, Pennington R, Ng J, Mendoza N: The time course of visual field recovery following transphenoidal surgery for pituitary adenomas: predictive factors for a good outcome. J Neurol Neurosurg Psychiatry 76:415-419, 2005

10. Jakobsson KE, Petruson B, Lindblom B: Dynamics of visual improvement following chiasmal decompression. Quantita- 
tive pre- and postoperative observations. Acta Ophthalmol Scand 80:512-516, 2002

11. Kerrison JB, Lynn MJ, Baer CA, Newman SA, Biousse V, Newman NJ: Stages of improvement in visual fields after pituitary tumor resection. Am J Ophthalmol 130:813-820, 2000

12. Lerner A, Mogensen MA, Kim PE, Shiroishi MS, Hwang DH, Law M: Clinical applications of diffusion tensor imaging. World Neurosurg 82:96-109, 2014

13. Lober RM, Guzman R, Cheshier SH, Fredrick DR, Edwards MS, Yeom KW: Application of diffusion tensor tractography in pediatric optic pathway glioma. J Neurosurg Pediatr 10:273-280, 2012

14. Nimsky C: Fiber tracking -we should move beyond diffusion tensor imaging. World Neurosurg 82:35-36, 2014

15. Paterno' V, Fahlbusch R: High-Field iMRI in transsphenoidal pituitary adenoma surgery with special respect to typical localization of residual tumor. Acta Neurochir (Wien) 156:463-474, 2014

16. Powell M: Recovery of vision following transsphenoidal surgery for pituitary adenomas. Br J Neurosurg 9:367-373, 1995

17. Roosendaal SD, Geurts JJ, Vrenken H, Hulst HE, Cover KS, Castelijns JA, et al: Regional DTI differences in multiple sclerosis patients. Neuroimage 44:1397-1403, 2009

18. Salmela MB, Cauley KA, Andrews T, Gonyea JV, Tarasiewicz I, Filippi CG: Magnetic resonance diffusion tensor imaging of the optic nerves to guide treatment of pediatric suprasellar tumors. Pediatr Neurosurg 45:467-471, 2009

19. Salminen LE, Conturo TE, Laidlaw DH, Cabeen RP, Akbudak E, Lane EM, et al: Regional age differences in gray matter diffusivity among healthy older adults. Brain Imaging Behav [epub ahead of print], 2015

20. Sherbondy AJ, Dougherty RF, Napel S, Wandell BA: Identifying the human optic radiation using diffusion imaging and fiber tractography. J Vis 8:12.1-12.11, 2008

21. Staempfli P, Rienmueller A, Reischauer C, Valavanis A, Boesiger P, Kollias S: Reconstruction of the human visual system based on DTI fiber tracking. J Magn Reson Imaging 26:886-893, 2007

22. Stieglitz LH, Lüdemann WO, Giordano M, Raabe A, Fahlbusch R, Samii M: Optic radiation fiber tracking using anteriorly angulated diffusion tensor imaging: a tested algorithm for quick application. Neurosurgery 68:1239-1251, 2011

23. Sun HH, Wang D, Zhang QJ, Bai ZL, He P: Magnetic resonance diffusion tensor imaging of optic nerve and optic radiation in healthy adults at 3T. Int J Ophthalmol 6:868-872, 2013
24. Taoka T, Sakamoto M, Nakagawa H, Nakase H, Iwasaki S, Takayama K, et al: Diffusion tensor tractography of the Meyer loop in cases of temporal lobe resection for temporal lobe epilepsy: correlation between postsurgical visual field defect and anterior limit of Meyer loop on tractography. AJNR Am J Neuroradiol 29:1329-1334, 2008

25. Trip SA, Wheeler-Kingshott C, Jones SJ, Li WY, Barker GJ, Thompson AJ, et al: Optic nerve diffusion tensor imaging in optic neuritis. Neuroimage 30:498-505, 2006

26. Tournier JD, Mori S, Leemans A: Diffusion tensor imaging and beyond. Magn Reson Med 65:1532-1556, 2011

27. Wu W, Rigolo L, O’Donnell LJ, Norton I, Shriver S, Golby AJ: Visual pathway study using in vivo diffusion tensor imaging tractography to complement classic anatomy. Neurosurgery 70 (1 Suppl Operative):145-156, 2012

28. Yamamoto A, Miki Y, Urayama S, Fushimi Y, Okada T, Hanakawa T, et al: Diffusion tensor fiber tractography of the optic radiation: analysis with 6-, 12-, 40-, and 81-directional motion-probing gradients, a preliminary study. AJNR Am J Neuroradiol 28:92-96, 2007

29. Yan Y, Li L, Preuss TM, Hu X, Herndon JG, Zhang X: In vivo evaluation of optic nerve aging in adult rhesus monkey by diffusion tensor imaging. Quant Imaging Med Surg 4:43-49, 2014

30. Zhang Y, Wan SH, Wu GJ, Zhang XL: Magnetic resonance diffusion tensor imaging and diffusion tensor tractography of human visual pathway. Int J Ophthalmol 5:452-458, 2012

\section{Disclosures}

The authors report no conflict of interest concerning the materials or methods used in this study or the findings specified in this paper.

\section{Author Contributions}

Conception and design: Hajiabadi, Fahlbusch. Acquisition of data: Hajiabadi. Analysis and interpretation of data: Hajiabadi, Fahlbusch. Drafting the article: Hajiabadi. Critically revising the article: Fahlbusch. Reviewed submitted version of manuscript: Hajiabadi. Approved the final version of the manuscript on behalf of all authors: Hajiabadi. Statistical analysis: Hajiabadi. Study supervision: Samii, Fahlbusch.

\section{Correspondence}

Mohamadreza Hajiabadi, International Neuroscience Institute, Rudolf-Pichlmayr Str. 4, Hannover 30625, Germany. email: dr.m.haji55@gmail.com. 